\title{
Statins for primary prevention: problems with cardiovascular-risk estimation?
}

Željko Reiner

I thank Yu-li Huang, Yi Huang, and Yan-xian $\mathrm{Wu}$ for their correspondence (Statins for primary prevention: identifying low-risk individuals. Nat. Rev. Cardiol.

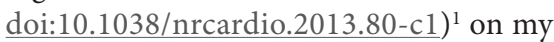
Review (Statins in the primary prevention of cardiovascular disease. Nat. Rev. Cardiol. doi:10.1038/nrcardio.2013.80). ${ }^{2}$ Huang and colleagues raise some interesting questions. I am glad that they agree that the use of statins for primary prevention in high-risk individuals is undoubtedly beneficial, and that the use of these drugs in low-risk individuals is still controversial. Risk estimation was not the main topic of the Review; nevertheless, in their correspondence, Huang et al. suggest that additional riskstratification options are needed to guide the use of statins in low-risk individuals. They propose that the level of C-reactive protein measured by high-sensitivity assay (hs-CRP) and the coronary artery calcium (CAC) score might be used to improve risk assessment in these individuals.

Total-cholesterol and LDL-cholesterol levels are associated with cardiovascular disease (CVD) risk in an independent and incremental manner and, therefore, lowering of the LDL-cholesterol level is the primary objective of dyslipidaemia management. ${ }^{3}$ These parameters also fulfil all the criteria for causality. The HDLcholesterol level can also contribute substantially to risk estimation, ${ }^{4}$ whereas other markers of lipid risk, such as the triglyceride and lipoprotein(a) levels, and some other biomarkers of CVD, do not. ${ }^{5-8}$ Even less useful for risk estimation is the hs-CRP level, which is associated with CVD risk, but also with death from several types of cancer and lung disease. The pathophysiological relevance of CRP to such a broad range of diseases is unclear. ${ }^{9}$ The association between the hs-CRP level and CVD has been shown to depend considerably on conventional CVD risk factors and other markers of inflammation. A lack of both specificity and a dose-effect or causality relationship exists between hs-CRP level and CVD risk. Therefore, the authors of the latest European guidelines on CVD prevention recommend that the hs-CRP level can be measured as a part of refined risk assessment only in patients with an unusual or moderate CVD risk profile, and even this recommendation is classified as class IIb, level of evidence B, and GRADE (Grading of Recommendations Assessment, Development and Evaluation) quality of evidence 'weak. ${ }^{10}$ Additionally, because of the previously mentioned shortcomings, the authors of the guidelines clearly recommend that the hs-CRP level should not be measured in asymptomatic, low-risk individuals or high-risk patients to assess 10-year risk of CVD.

Regarding the prognostic value of the CAC score in asymptomatic, low-risk individuals, CAC assessment might be useful in intermediate-risk or moderate-risk (but not low-risk) individuals, in whom the required intensity of risk-factor modification, including statin treatment, is often uncertain. ${ }^{11-13}$ In these individuals, a high CAC score might help to identify those at high risk, in whom intensive treatment of risk factors, including dyslipidaemia, might be warranted; by contrast, the absence of CAC would reclassify these individuals to the low-risk group. ${ }^{14}$ However, the percentage of moderate-risk individuals that would be reclassified using CAC scoring remains unknown. Therefore, the authors of the European guidelines on CVD prevention recommend that CAC scoring should be considered for CVD risk assessment only in moderate-risk individuals, and even this recommendation is classified as class IIa, level of evidence B, and GRADE quality of evidence 'weak.. ${ }^{10}$

Department of Internal Medicine, University Hospital Centre Zagreb, School of Medicine, University of Zagreb, Kispaticeva 12, 10000 Zagreb, Croatia. zreiner@kbc-zagreb.hr
Competing interests

The author declares associations with the following companies: Abbot, AstraZeneca, Bayer, and Sanofi. See the article online for full details of the relationships.

1. Huang, Y.-I., Huang, Y. \& Wu, Y.-x. Statins for primary prevention in individuals at low cardiovascular risk. Nat. Rev. Cardiol. http:// dx.doi.org/10.1038/nrcardio.2013.80-c1.

2. Reiner, Ž. Statins in the primary prevention of cardiovascular disease. Nat. Rev. Cardiol. http://dx.doi.org/10.1038/nrcardio.2013.80.

3. Graham, I., Cooney, M. T., Bradley, D., Dudina, A $\&$ Reiner, Ž. Dyslipidemias in the prevention of cardiovascular disease: risks and causality. Curr. Cardiol. Rep. 14, 709-720 (2012).

4. Reiner, Ž. et al. ESC/EAS guidelines for the management of dyslipidaemias: the Task Force for the management of dyslipidaemias of the European Society of Cardiology (ESC) and the European Atherosclerosis Society (EAS). Eur. Heart J. 32, 1769-1818 (2011).

5. Chapman, M. J. et al. for the European Atherosclerosis Society Consensus Panel. Triglyceride-rich lipoproteins and high-density lipoprotein cholesterol in patients at high risk of cardiovascular disease: evidence and guidance for management. Eur. Heart J. 332 , 1345-1361 (2010).

6. Nordestgaard, B. G. et al. for the European Atherosclerosis Society Consensus Panel. Lipoprotein(a) as a cardiovascular risk factor: current status. Eur. Heart J. 23, 2844-2853 (2010).

7. Zannad, F. et al. Prevention of cardiovascular disease guided by total risk estimationschallenges and opportunities for practical implementation: highlights of a CardioVascular Clinical Trialists (CVCT) Workshop of the ESC Working Group on CardioVascular Pharmacology and Drug Therapy. Eur. J. Prev. Cardiol. 19, 1454-1464 (2012).

8. Zannad, F. et al. Risk stratification in cardiovascular disease primary preventionscoring systems, novel markers, and imaging techniques. Fundam. Clin. Pharmacol. 26 , 163-174 (2012).

9. Kaptoge, S. et al. C-reactive protein concentration and risk of coronary heart disease, stroke, and mortality: an individual participant meta-analysis. Lancet $\mathbf{3 7 5}$, 132-140 (2010).

10. Perk, J. et al. European guidelines on cardiovascular disease prevention in clinical practice (version 2012). Eur. Heart J. 33 , 1635-1701 (2012).

11. Graham, I. et al. European guidelines on cardiovascular disease prevention in clinical practice: executive summary. Eur. Heart J. 28, 2375-2414 (2007). 


\section{CORRESPONDENCE}

12. Grundy, S. M. et al. Implications of recent clinical trials for the National Cholesterol Education Program Adult Treatment Panel III guidelines. Circulation 110, 227-239 (2004).

13. van Werkhoven, J. M. et al. Multislice computed tomography coronary angiography for risk stratification in patients with an intermediate pretest likelihood. Heart 95, 1607-1611 (2009).
14. Perrone-Filardi, P. et al. Cardiac computed tomography and myocardial perfusion scintigraphy for risk stratification in asymptomatic individuals without known cardiovascular disease: a position statement of the Working Group on Nuclear Cardiology and Cardiac CT of the European Society of Cardiology. Eur. Heart J. 32, 1986-1993 (2011). 